\title{
Sex ratio of newborn infants born to pregnant women with severe chronic constipation
}

This article was published in the following Dove Press journal:

Clinical Epidemiology

9 September 2010

Number of times this article has been viewed

\author{
Andrew E Czeizel' \\ Erzsébet H Puhól \\ Ferenc Bánhidy² \\ 'Foundation for the Community \\ Control of Hereditary Diseases, \\ ${ }^{2}$ Second Department of Obstetrics \\ and Gynecology, Semmelweis \\ University, School of Medicine, \\ Budapest, Hungary
}

\begin{abstract}
There was a significant male excess in the newborns of pregnant women with severe chronic constipation during pregnancy compared to pregnant women without constipation and pregnant women with new onset severe constipation, during pregnancy.
\end{abstract}

Keywords: constipation, pregnancy, birth outcomes, sex ratio, male excess

Constipation affects between $11 \%$ and $38 \%$ of pregnant women; ${ }^{1}$ the aim in our previous study was to estimate the association between pregnant women with constipation and congenital abnormalities in their offspring ${ }^{2}$ in the Hungarian Case-Control Surveillance of Congenital Abnormalities (HCCSCA), 1980-1996. ${ }^{3}$ Here, an unexpected secondary finding of the study is reported.

The HCCSCA is based on the comparison of cases with different congenital abnormalities and matched controls (ie, newborns without any defect). Cases are selected from the Hungarian Congenital Abnormality Registry while controls are collected from the National Birth Registry of the Central Statistical Office. In general two controls are matched to each case according to sex, date of birth, and area of residence of mothers. Here only the data of controls are shown because congenital abnormalities may have a more drastic effect for birth outcomes than maternal constipation. Prenatal care was mandatory for pregnant women in Hungary, the average number of their visits was seven during the study pregnancy and obstetricians recorded all maternal diseases and related drug treatments in a prenatal maternity logbook. In addition mothers were asked to fill in a structured questionnaire regarding their diseases and related drug treatments during the study pregnancy after their deliveries. The birth outcomes of newborns were recorded in the discharge summaries (in Hungary almost all deliveries occurred in inpatient obstetric clinics).

Statistical analyses were carried out using SAS version 8.02 (SAS Institute Ins., Cary, NC). The categorical variables of mothers with and without severe constipation as reference group and their newborns were compared by chi-square test and odds ratio (OR) with $95 \%$ confidence intervals $(\mathrm{CI})$ in ordinary logistic regression model, while the Student's $t$-test was used to analyze quantitative variables.

The preliminary analysis of pregnant women with constipation showed two different categories: (1) Severe constipation was recorded in the prenatal maternity logbooks and these pregnant women were treated with drugs (generally by senna, rarely by phenolphthalein); and (2) Mild constipation was reported retrospectively in the questionnaire by mothers, and in general these pregnant women were not treated
Correspondence: Andrew E Czeize of Hereditary Diseases, 1026 Budapest, Törökvész lejtõ 32. Hungary Email czeizel@interware.hu 
with laxative drugs. The maternal sensitivity and reporting attitude was different, particularly regarding nonsevere pathological symptoms. ${ }^{3}$ Thus we decided to evaluate only pregnant women with severe constipation in the study. Of 38,151 controls, 144 mothers had severe constipation and these women were older than controls (mean age \pm standard deviation (SD): $26.6 \pm 4.9$ versus $25.4 \pm 4.9$ years) with a higher proportion of professional and managerial employment (50.0\% versus 37.9\%), and with more frequent use of folic acid during the study (64.6\% versus $54.4 \%)$ compared to 38,007 pregnant women without severe constipation.

The birth outcomes of newborns were also compared in the groups of pregnant women with or without severe constipations: a longer mean gestational period $(39.8 \pm 1.9$ versus $39.4 \pm 2.2$ weeks, $P=0.03$ ) and lower rate of preterm birth $(4.2 \%$ versus $9.2 \%$, OR with $95 \%$ CI: 0.46 , $0.21-0.96$ ) were found in the newborns of women with severe constipation. However, the mean birth weight $(3,275 \pm 516$ versus $3,276 \pm 511 \mathrm{~g}, P=0.11)$ and the rate of low birth weight (4.2\% versus 5.7\%, OR with 95\% CI: 1.14, 0.43-2.93) did not show significant difference in these two groups of newborns. The lower rate of preterm birth can be explained by the more frequent use of folic acid by the mothers with severe constipation. ${ }^{4}$
The major unexpected finding was an obvious male excess in the newborns of pregnant women with severe constipation (Figure 1). At first sight the male excess in the newborns of reference pregnant women without medically recorded constipation is disturbing. The explanation is that controls in the HCCSCA were matched to the cases according to sex and cases with congenital abnormalities have an obvious male excess (65\%:35\%) in the HCCSCA. This well-know male excess among malformed cases is explained by some X-linked defects (eg, some part of cleft palate), by certain congenital abnormalities such as congenital pyloric stenosis, and mainly by a much higher incidence of defects in male genital organs (hypospadias and undescended testis) than in female genital organs.

In the next step two types of severe constipation were differentiated (Figure 1): chronic (the onset was before conception which continued during pregnancy) and new-onset (after the third gestational months). The male excess was significantly higher only in the newborns of pregnant women with severe chronic constipation $\left(\chi_{1}^{2}=5.9, P=0.01\right)$.

Theoretically there are four explanations for this unexpected finding: (i) A higher rate of miscarriages (ie, early postconceptional selection) of female embryos. Unfortunately the HCCSCA is not appropriate for the evaluation of

\section{Total number of newborns}

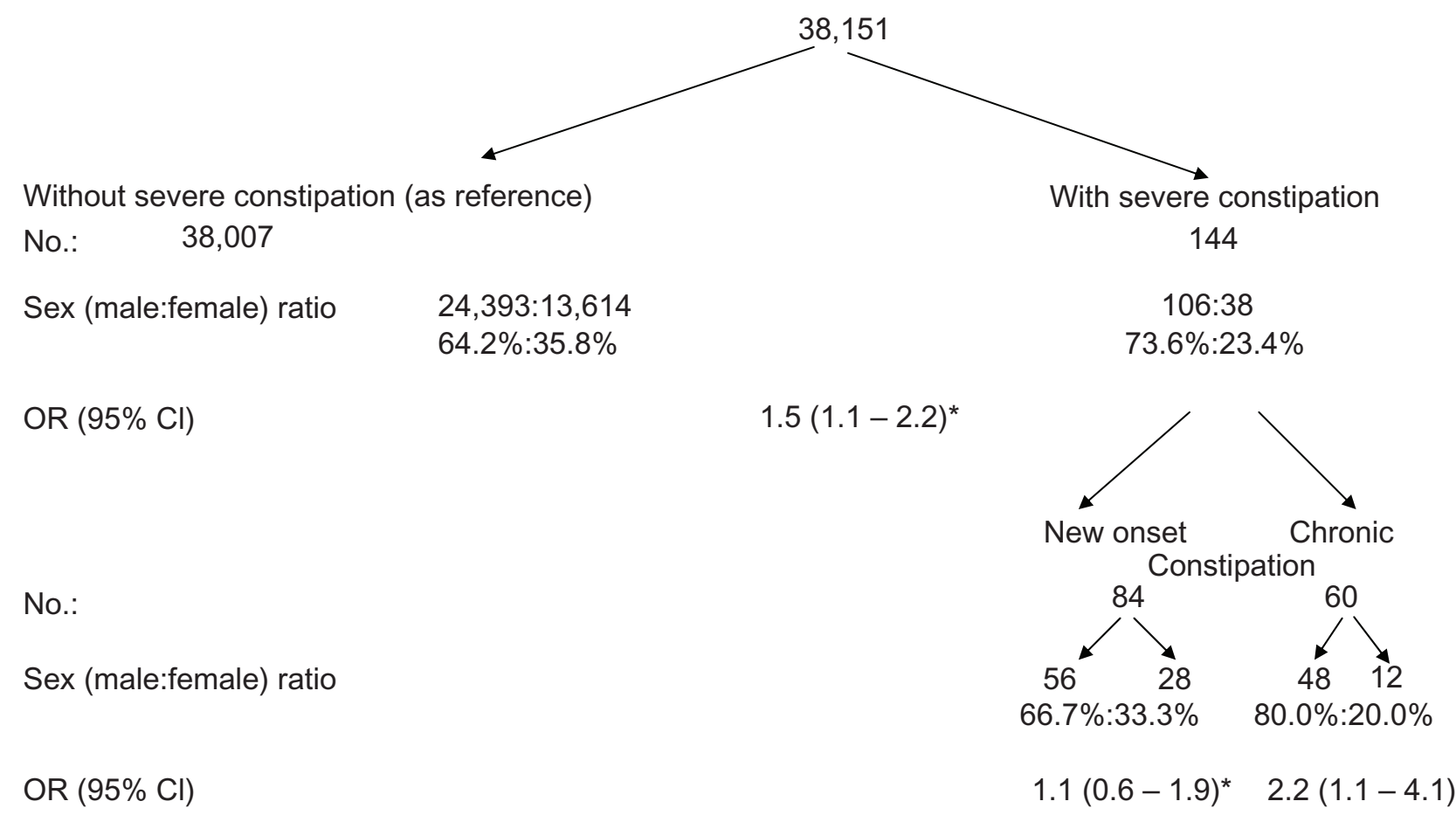

Figure I Data set of control newborns in the HCCSCA, 1980-1996.

Notes: *Adjusted for maternal age and employment, in birth order.

Abbreviations: HCCSCA, Hungarian Case-Control Surveillance of Longenital Abnormalities; CL, confidence intervals; OR, odds ratio. 
miscarriages in the study pregnancy. However, the rate of miscarriages is known in the previous pregnancies of these women, and it was not higher, though we could assume that the chronic constipation existed in their previous pregnancies as well; (ii) The sex of conceptions is determined mainly by the sex chromosome of spermatozoa, ie, males. Pregnant women are in the focus of the HCCSCA, although assessments of the major diseases in the fathers of cases and controls are included in the questionnaire. There was no cluster of specific diseases in the male partners of pregnant women with severe chronic constipation; (iii) Chronic severe constipation may modify the $\mathrm{pH}$ of vaginal fluid and the less acidic vaginal $\mathrm{pH}$ may favor the transfer of spermatozoa including Y chromosome; ${ }^{5}$ (iv) Results could be due to chance or unevaluated confounders.

In conclusion, severe chronic constipation of pregnant women was found to be associated with a higher proportion of males among newborns. This finding is not important from a clinical perspective, but theoretically is an interesting phenomenon therefore further studies are required.

\section{Disclosure}

The authors report no conflicts of interest in this work.

\section{References}

1. Williamson C. Gastrointestinal disease. Clinical Obstet Gynaecol. 2001; 15:937-952.

2. Ács N, Bánhidy F, Puhó HE, Czeizel AE. No association between severe constipation with related drug treatment in pregnant women and congenital abnormalities in their offspring: a population-based case-control study. Cong Anom. 2009;49:157-168.

3. Czeizel AE, Rockenbauer M, Siffel CS, Varga E. Description and mission evaluation of the Hungarian Case-Control Surveillance of Congenital Abnormalities, 1980-1996. Teratology. 2001;63:176-185.

4. Czeizel AE, Puho EH, Langmar Z, Ács N, Bánhidy F. Possible association of folic acid supplementation during pregnancy with reduction of preterm birth: a population-based study. Eur J Obstet Gynecol Reprod Biol. 2010; 148:135-140.

5. Sills ES, Kirman I, Thatcher SS III, Palermo GD. Sex-selection of human spermatozoa: evolution of current techniques and applications. Arch Gynec Obstet. 1998;261:109-115.
Clinical Epidemiology

\section{Publish your work in this journal}

Clinical Epidemiology is an international, peer-reviewed, open access journal focusing on disease and drug epidemiology, identification of risk factors and screening procedures to develop optimal preventative initiatives and programs. Specific topics include: diagnosis, prognosis, treatment, screening, prevention, risk factor modification, systematic

\section{Dovepress}

reviews, risk \& safety of medical interventions, epidemiology \& biostatical methods, evaluation of guidelines, translational medicine, health policies \& economic evaluations. The manuscript management system is completely online and includes a very quick and fair peer-review system, which is all easy to use. 